\title{
De G. Politzer et sa critique de l'inconscient de Freud au a-réalisme de J. Lacan
}

\section{From G. Politzer and his critique of Freud's unconscious to J. Lacan's a-realism}

\author{
Mélinda Marx*1 \\ Nelson da Silva Junior*2 \\ Jean-Luc Gaspard*3
}

En 1928, dans sa "Critique des Fondements de la Psychologie », $G$. Politzer esquisse les traits d'une psychologie qui vise à réformer la psychologie expérimentale universitaire. S'appuyant sur la théorie des rêves de Freud, Politzer présente une conception de la psychologie tout à fait inédite à son époque. Lacan s'inspirera de ses travaux pour sa thèse sur la paranoïa, participant d'un débat violent au sein du mouvement communiste partagé entre anti-freudisme et freudo-marxisme. En donnant une place essentielle à la parole et au récit, Politzer revendiquait une psychologie concrète. Son refus de la doctrine freudienne, selon lui tributaire de la tradition métaphysique de la psychologie, incluait aussi l'hypothèse de l'inconscient dans une sévère critique du réalisme, inclusion qui ne sera pourtant jamais accepté par Lacan qui s'y opposera avec la prémisse que l'inconscient est structuré comme un langage. Cette orientation allait en toute logique imposer un réalisme de la structure qui s'articule, à son tour, à la faille de la cause empirique.

Mots clés: Psychologie concrète, inconscient, réalisme, objet $a$

*1,3 Université de Rennes 2 (Rennes, França).

*2 Universidade de São Paulo - USP (São Paulo, SP, Brasil). 
S'il y a quelque chose que je suis, il est clair que je ne suis pas nominaliste. Je veux dire que je ne pars pas de ceci que le nom est quelque chose qui se plaque, comme ça sur du réel. [...] Il ne s'agit pas d'être réaliste

[...] au sens du réalisme des universaux, mais il s'agit de pointer ceci, que notre discours, notre discours scientifique, ne trouve le réel qu'à ce qu'il dépend de la fonction du semblant. L'articulation, j'entends algébrique, du semblant - et comme tel il ne s'agit que de lettre - et ses effets, voilà le seul appareil au moyen de quoi nous désignons ce qui est réel. Ce qui est réel, c'est ce qui fait trou dans ce semblant, dans ce semblant articulé qu'est le discours scientifique. (J. Lacan, 2006b, p. 28).

\section{Introduction}

En 1946, dans ses "Propos sur la causalité psychique », J. Lacan souligne l'enjeu, voire l'urgence de dégager le concept de science en psychologie. Il pose la nécessité de construction d'un espace rationnel qui permette d'appréhender de façon " concrète $»$ le « complexe spatio-temporel imaginaire » qui lie le sujet à sa cause (Lacan, 1946/1966b, p. 188). Dans ce texte, Lacan rappelle la contribution majeure de G. Politzer à cette question dans sa Critique des fondements de la psychologie $e^{1}$ publié en 1928. Toutefois, malgré

${ }^{1}$ Dorénavant référé simplement comme « $\mathrm{CFP}$ » 
la rareté avec laquelle le psychanalyste en fait mention, il est assez connu aujourd'hui que son projet est hautement débiteur des critiques que le philosophe avait adressées, en son temps, aux psychologues quant au statut scientifique de leur objet. Une critique qui visait alors à réformer tout l'édifice conceptuel de la psychologie classique, en même temps qu'elle donnait les prémisses à la constitution d'une psychologie concrète dont Politzer cherchait à établir les conditions de possibilité épistémologiques. Or, il est notable que, dès sa thèse de doctorat, le jeune Lacan, confronté aux difficultés de présenter une fondation psychologique épistémologiquement solide et à la hauteur de la clinique psychiatrique qu'il se proposait de renouveler, n'a apparemment pas trouvé un meilleur appui que celui offert par la CFP de Politzer (Simanke, 2002, pp. 160-165). Malgré cela, et en dépit du fait de l'appel à la « concrétude » qui ponctue le texte de sa thèse, le nom de Politzer n'est à aucun moment prononcé dans celle-ci. Peu à peu, ce nom apparaîtra dans ses textes ultérieurs, ce qu'il fera pourtant avec parcimonie, sans jamais pour autant rendre véritablement justice à l'ampleur et à l'importance stratégique que ce texte séminal de Politzer ${ }^{2}$ avait pris dans sa pensée. Profondément influencé par le matérialisme de K. Marx, Politzer y souligne l'importance de penser l'homme comme un être essentiellement social, avec toutes les conséquences que cette prise de position implique pour n'importe quelle science de l'homme, la psychologie en première ligne. «La philosophie a besoin d'une matière réelle pour être valable dans l'esprit »(Politzer, 1926/1969a, p. 22). Or, " l'Homme est seul avec sa pensée » (Ibid., p. 43), mais il ne le sait pas, et c'est à l'ignorer qu'il traite « ses affaires les plus personnelles sur un plan qui l'est le moins possible, sur le plan cosmologique, sur le plan théologique, sur le plan de l'absolu, sur tous les plans, sauf sur celui qui est véritablement le sien, c'està-dire le plan humain » (Ibid., p. 42). Ainsi, dès 1926, on entend déjà, dans cette sentence, la dénonciation de l'expectative de neutralité qu'il fera, par

${ }^{2}$ Né en 1903 en Hongrie, G. Politzer quitte son pays en 1920 après la défaite de Béla Kun. Passant par l'Autriche, Politzer suit quelques mois les séminaires de la société psychanalytique où il rencontre S. Freud et S. Ferenczi. En 1921, il s'installe à Paris et obtient l'agrégation de philosophie. En 1924, il participe à la création de la revue Philosophies aux côtés de P. Morhange, N. Guterman, G. Friedman et H. Lefevbre. Il s'opposera tout particulièrement aux cadres traditionnels de la philosophie représentés par L. Brunschvicg et H. Bergson dans une série d'écrits publiés entre 1924 et 1939. En 1926, Politzer écrit un petit texte d'Introduction visant à dégager les prémisses d'une philosophie concrète. 


\section{EPISTEMOLOGIA DA PSICOPATOLOGIA}

la suite, aussi bien à la psychologie introspective qu'à celle expérimentale. Pour Politzer, ces deux versants de la psychologie s'unissent en effet dans la même erreur, notamment celle de ne considérer l'homme qu'à partir d'une situation abstraite, irréelle, introuvable dans la vie et le monde social qui sont les siens. Cette critique proprement épistémologique se dédouble dans une lecture de l'histoire de la pensée, où la métaphysique est comprise comme une héritière de la soumission religieuse du croyant à Dieu : «L'humanité se donne aux forces qu'elle croit réelles; elle se soumet et se laisse manœuvrer ; elle se laisse martyriser et détruire en croyant qu'il y a là une nécessité inéluctable et en quelque sorte, cosmique, par cet acte de soumission, elle devient matière ; malléable sans remords et sans sympathie, au même titre que l'airain ou la terre. Une civilisation l'entoure dont toutes les institutions n'ont pour but que de le maintenir dans cet état de matière. L'homme meurt et ne sait pas.» (Ibid., p. 45). Dans le même élan, les écrits de Politzer témoignent d'une volonté du philosophe de mettre en question les principes fondateurs de la psychologie. Sa critique est réformatrice et vise un dépassement de la querelle entre psychologie subjective ou introspective et psychologie expérimentale ou objective. Puisque ces deux versants à l'époque étaient ceux qui tenaient les titres de dignité à l'académie, son projet, lorsqu'il tient à une restitution intégrale des problématiques de la psychologie sur d'autres fondements, ne pouvait être que révolutionnaire, aussi bien du point de vue conceptuel pour cette discipline que vis-à-vis des structures académiques de l'université française. Dans ses projets, la Critique des fondements de la psychologie était le premier livre d'une œuvre qui devait en compter trois, regroupés sous le titre Matériaux eux-mêmes, posés comme préliminaire à un Essai critique sur les fondements de la psychologie. Les deux autres livres devaient traiter de la théorie de la Gestalt et de la phénoménologie pour l'un ; du behaviorisme, pour l'autre. Son entreprise restera toutefois inachevée.

De son interprétation des concepts freudiens, Politzer va, le premier, extraire ce que " les historiens de la psychanalyse qualifient de lecture herméneutique de Freud, par opposition à ce qui serait qualifié de lecture positiviste, qui met l'accent sur ses aspects biologisants et déterministes » (Furlan, 1999, p. 119). Une lecture qui va imposer à la fortune critique de l'œuvre freudienne et durant de longues périodes une dualité entre signification et sens (Askofaré, Gaspard, Macary-Garipuy \& Sauret, 2008), si bien cristallisée dans le grand livre de Ricoeur, De l'interprétation : essai sur Freud (1995). A ces deux courants, l'on en rajoutera un troisième : 
[...] la lecture de Lacan, type de lecture déjà détectée par Foucault dans Les mots et les choses (1966), et qui plaçait la psychanalyse, à côté de la linguistique, dans un autre espace épistémologique que celui occupé par les philosophies de la finitude tels Heidegger et Merleau-Ponty. (Ibid., p. 119)

Toutefois, selon Prado Jr., l'influence soutenue de cet écrit annoncé comme préliminaire par Politzer lui-même, apparaît somme toute énigmatique, au vu de l'échec des travaux ultérieurs de ce dernier à susciter le même intérêt (Prado Jr., 1991, p. 14). Énigme qui, cependant, peut être comprise si l'on tient compte d'une triple position méthodologique vis-à-vis $\mathrm{du}$ récit, position aussi présente chez M. Merleau-Ponty et J-P. Sartre dans une période de plein essor de la phénoménologie : tout d'abord le récit centré sur la première personne; ensuite, le récit conçu comme méthode, à laquelle le psychanalyste S. Leclaire donnera toute son importance dans le débat qui l'opposera à Lacan ; enfin, le récit associé à l'intentionnalité de l'action. Ces trois aspects, au demeurant hétérogènes, auraient pu, dans chacun de ces cas, garantir l'importance de la Critique des fondements de la psychologie CFP (Prado Jr., 1991, p. 19).

\section{S'orienter du concret}

\section{Critique du réalisme}

« L'avènement de la psychologie « expérimentale », loin de représenter un nouveau triomphe de l'esprit scientifique, n'en était qu'une humiliation» (Politzer, 2003, p. 7). Dans une critique acerbe, Politzer vise à montrer les impasses de la psychologie classique en même temps que sa survivance au sein de théories qui s'étaient constituées contre elle. Ces impasses, il les situe en son fondement même, à savoir le christianisme et son " culte de l'âme ». $\mathrm{Au}$ fond, il y aurait une récupération de cette entité aussi bien sur le versant de l'introspection que celui expérimental de la psychologie. Toutes deux se seraient égarées d'avoir réduit leur objet à une abstraction de l'humain, lui ôtant ses déterminations sociales et historiques. Et, même si la critique kantienne a éliminé la notion d'âme, la psychologie classique n'aurait cependant survécu qu'en se déguisant en la modalité d'une science à la mode au XIXème, soit celle de l'expérience et du calcul. C'est cet argument qui permet à Politzer de lancer sa fameuse devise : " les psychologues sont scientifiques comme les sauvages évangélisés sont chrétiens » ( Ibid., p. 8). 


\section{EPISTEMOLOGIA DA PSICOPATOLOGIA}

En guise de modèle de ce qui serait une psychologie concrète, capable d'éviter les écueils de ce piège vétuste, Politzer s'appuie sur le moment inaugural de la psychanalyse, la Traumdeutung (Freud, 1900/2003). Ainsi, là où la psychologie classique conçoit le rêve comme un phénomène organique, un déchet de l'esprit ou une construction fortuite, pour la lecture que Politzer en fait de Freud, il est une modalité de la manifestation du désir, soit un élément de la vie psychique inséparable du sujet en situation. Or selon Politzer, la démarche d'abstraction de la psychologie classique fait que le rêve et le sujet s'en trouvent déliés l'un de l'autre. Le rêve devient «une collection d'états en soi » (Ibid., p. 38), « suspendu dans le vide » (Ibid., p. 39). La psychanalyse donne ainsi la direction d'une nouvelle définition du fait psychologique, non plus attachée à une démarche d'abstraction comme ce sont les cas et de psychologie introspective et de celle de laboratoire, mais orientée vers le concret, un concret incarné.

Ceci dit, la psychologie ne peut être, à son avis, qu'une science empirique fondée sur l'induction, et le «Je » ne peut être que l'individu singulier envisagé dans sa dimension dramatique, dans le sens dialogique de ce terme. Ainsi, il souligne l'incompatibilité de ce «Je » avec le « Je » transcendantal de Kant. En effet, si Kant satisfait à l'exigence de la première personne et de l'homogénéité avec sa théorie de la synthèse, son étude exclue cependant l'expérience concrète. Le « Je » de Politzer n'est pas le sujet d'un acte transcendantal, mais, pour répondre à l'exigence d'homogénéité, doit être situé au même plan que l'individu concret. Et si l'acte de l'individu concret est la vie de l'individu singulier, cette dernière n'est pas qualitative mais dramatique, au sens qu'elle implique l'interaction avec ses semblables dans sa structure. Les faits psychologiques vont dès lors être considérés comme « les segments de la vie de l'individu singulier » (Ibid., p. 51).

Politzer s'oppose ainsi à la démarche d'abstraction de la psychologie classique qui vise à remplacer le drame personnel par un drame impersonnel, « purement notionnel » (Ibid., p. 52). C'est ce qui constitue l'une de ses principales critiques à la psychologie classique, soit que celle-ci cherche à étudier les faits de la première personne en troisième personne pour atteindre à son statut de science. Or, suivant Politzer, ce véritable tour de force, ce « miracle » ne peut être réalisé que parce que cette psychologie s'appuie sur le Réalisme et devient une sorte de "paraphysique » décrivant un monde spirituel placé en parallèle du monde physique. "Les psychologues ne se sont pas aperçus qu'ôter le je aux faits psychologiques, c'est les anéantir » (Ibid., p. 46) et que toute démarche fondée sur l'abstraction ne peut être que « fabulation ». 
La psychologie classique est « l'élaboration notionnelle d'un mythe » (Ibid., p. 8). Au contraire, avec la technique de l'interprétation, Freud « ne cherche pas un scénario abstrait et impersonnel dont les figurants sont des excitations physiologiques, et dont l'intrigue est constituée par leur promenade dans les cellules cérébrales » (Ibid., p. 53). L'interprétation de Freud vise non pas un moi abstrait mais « le moi de la vie quotidienne » (Ibid., p. 53). Un moi qui n'est pas « propriétaire de ses états » ou « cause d'une fonction générale », mais est " agent d'un acte considéré dans sa détermination singulière » (Ibid., p. 53). Ainsi, le rêve sera envisagé comme une « modulation du je » et le désir devient ce qui lui assure une « continuité du je » (Ibid., p. 39).

\section{Critique de l'inconscient}

La psychologie classique, avec la technique de l'introspection, est conduite à faire abstraction de l'intention significative. Dès lors, elle ne s'intéresse pas tant à ce que le sujet raconte, qu'à ce qui se passe dans son esprit pendant qu'il parle. La psychologie classique dédouble le récit et fait de son double une réalité interne qu'elle décrit dans un formalisme fonctionnel. A suivre Politzer, en renouvelant la définition du fait psychologique, la psychanalyse a pu abandonner l'investigation de la réalité intérieure au profit de l'analyse du drame. Une " attitude concrète » qui ne trouvera toutefois que des débouchés explicatifs décevants dans la théorie de Freud. "Tout se passe comme si Freud voulait, par ses explications, refaire en sens inverse le chemin que l'inspiration concrète de la psychanalyse lui a fait parcourir et il veut, en quelque sorte, se faire pardonner ses découvertes concrètes en en donnant une explication dans le goût de la psychologie classique » (Politzer, 2003, p. 111). L'antagonisme entre psychologie abstraite et psychologie concrète que Politzer avait dégagé entre psychologie classique et psychanalyse, se trouve redoublé à l'intérieur même de la psychanalyse. Autrement dit, la psychanalyse se trouve traversée par ce double mouvement, l'un orienté vers le concret, l'autre vers l'abstraction. Ainsi, l'interprétation s'inscrit dans l'inspiration concrète de donner toute sa valeur au récit, en tant que matériel objectif et significatif. Mais l'hypothèse de l'inconscient vient contrebalancer cette orientation vers le concret. L'introduction de l'hypothèse de l'inconscient résulte « de la persistance à l'intérieur de la théorie freudienne, des exigences et des démarches fondamentales de la psychologie abstraite » (Ibid., p. 156). L'hypothèse de l'inconscient ne peut ainsi être posé que dans une exigence réaliste à laquelle s'adjoint un formalisme fonctionnel. Pour Politzer, Freud confond l'empirique 


\section{EPISTEMOLOGIA DA PSICOPATOLOGIA}

et le conceptuel et rejoint une position réaliste qui, au lieu de s'en tenir à la signification, "cherche une entité psychique à réaliser » (Ibid., p. 171). Dans cette opération, le récit perd sa valeur significative, mais s'illusionne cependant de donner quelque chose de nouveau. L'hypothèse de l'inconscient s'énonce tel que « le sujet pense plus qu'il ne croit penser, et son savoir avoué ne représente qu'un fragment de son savoir véritable » (Ibid., p. 160).

Pour Politzer, la dynamique du fait psychologique, c'est la dynamique du «Je », et la seule donnée réelle est le récit significatif. Le contenu de connaissance, le sens du rêve, n'a pas d'existence en dehors de la démarche réaliste. «Le sujet a rêvé : c'est tout ce qu'il avait à faire. Il ne connaît pas le sens du rêve : il n'a pas à le connaître en tant que sujet pur et simple, car cette connaissance regarde le psychologue ; bref, le contenu latent, c'est-à-dire la connaissance du sens du rêve, ne peut être avant l'analyse ni conscient, ni inconscient : il n'existe pas » (Ibid., p. 205). Ainsi que le terme de l'inconscient, ceux qui en sont les corrélats, comme celui de l'autre scène, seront aussi considérés par Politzer comme un concept qui rabat les données empiriques obtenues par les associations langagières du sujet sur un réalisme qui les dépouille de leur valeur significative. (Simanke, 2002, pp. 182-183).

\section{Des références discrètes chez Lacan}

Les références explicites de Lacan à Politzer sont rares, touches subtiles qui ne laissent pas présager, au premier abord, de l'importance que revêt la pensée du philosophe dans l'élaboration du psychanalyste. Dans un premier temps, l'emprunt à Politzer sert de jalon dans la valorisation de la méthode freudienne vis-à-vis de sa doctrine (Simanke, 2002, p. 161). C'est aussi une référence majeure mais néanmoins silencieuse dans l'article « La famille » écrit pour 1'Encyclopédie française avant d'être publié sous le titre « Les complexes familiaux dans la formation de l'individu » (Lacan, 1938/2001a) comme dans son article « Propos sur la causalité psychique » en 1946.

Dans sa thèse, Lacan travaille le cas Aimée à partir « d'une description concrète », permettant de rendre compte de la psychose par « une méthode théoriquement plus rigoureuse » (Lacan, 1932/1998, p. 15). S'opposant à une conception de la folie purement organique, Lacan va ainsi donner une place centrale au drame individuel comme à l'idée que la folie n'est pas déficitaire mais «phénomène de pensée » (Lacan, 1946/1966b, p. 162) : conception qui se retrouvera dans ses écrits ultérieurs, alors même que Lacan aura conquis son indépendance théorique, marquant irrémédiablement son approche de 
la folie de l'influence précoce de Politzer. En 1946, dans ses « Propos sur la causalité psychique ", Lacan reprendra les avancées théoriques de sa thèse de 1932, soulignant que " la folie est vécue, toute dans le registre du sens ». Il ajoutera : «Le phénomène de la folie n'est pas séparable du problème de la signification pour l'être en général, c'est-à-dire du langage pour l'homme » (Lacan, 1946/1966b, p. 166). Toute l'entreprise de Lacan, dans ces premières années, vise ainsi à dégager l'inconscient de tout ancrage biologique, dans la promotion du langage et de la parole. Et c'est avec la lecture de Politzer en tant qu'il promeut le récit, que Lacan va engager son « retour à Freud ». L'appel de Politzer à une psychologie qui s'en tienne aux limites concrètes de la situation de l'homme, soit le langage en général, et plus particulièrement la parole, en ce que sa dimension d'acte social reste irréductible aux conceptualisations qu'elle en peut subir, est ainsi particulièrement opérationnelle pour le premier Lacan.

Par ailleurs, Politzer se retrouvera aussi en arrière-fond de la référence de Lacan à Merleau-Ponty contre la perspective organo-dynamique de Henry Ey. En effet, la $C F P$ « nous présente une critique de l'œuvre freudienne qui a particulièrement intéressé Merleau-Ponty, et la référence à celle-ci ne pouvait manquer dans l'étude des relations de la pensée de Merleau-Ponty avec la psychanalyse ». (Furlan, 1999, p. 118). Si Merleau-Ponty se réfère explicitement à Binswanger, le vocabulaire utilisé n'en reste pas moins emprunté à Politzer, témoignant de ce double ancrage de sa conception entre existentialisme et concret. Un double ancrage qui se retrouvera dans la philosophie de Sartre, tout aussi marquée par la pensée de Politzer, qu'il accordera avec la phénoménologie de Husserl et la philosophie d'Heidegger. Lanteri-Laura soulignait ainsi l'apport majeur de Politzer et Nizan dont l'oeuvre « bien plus que toute autre, nous a aidés, après 1945, à assimiler la théorie de la forme et la phénoménologie. Sans eux, sans leur influence sur Merleau-Ponty et sur Sartre, nous aurions certainement mis dix ans de plus à tirer profit de la philosophie allemande contemporaine et c'est bien une dette que leur nouvelle lecture rend plus évidente » (Lantéri-Laura, 1968, p. 785).

Pour Lacan, il s'agit avant tout de s'opposer à une perspective causaliste du phénomène, pour l'envisager au niveau du sens qu'il prend pour un sujet. ${ }^{3}$ Toutefois, la question d'une primauté du sens dans la situation concrète de

${ }^{3}$ Prenons l'exemple qu'en donne Lacan dans son séminaire sur Les Psychoses (19551956/1981) : un enfant reçoit un coup et se demande : " est-ce une caresse ou une gifle ?" Si l'autre lui répond que c'est une claque, l'enfant pleure ; si au contraire, on lui dit que c'est une caresse, il est heureux (pp. 14-15). 


\section{EPISTEMOLOGIA DA PSICOPATOLOGIA}

l'humain, conduira à un tournant important dans l'économie conceptuelle de Lacan qui prend son essor à partir des années 50 influencées par la linguistique structuraliste. Ce tournant lui permettra de marquer ses différences d'avec Merleau-Ponty après cette période d'affinités théoriques. Car, si MerleauPonty manifeste de l'intérêt pour la psychanalyse dans son questionnement de l'intentionnalité, l'inconscient dont il parle dans La structure du comportement (Merleau-Ponty, 1942/2013) n'en reste pas moins très éloigné de la lecture de Lacan. A partir des années 50, les deux approches deviennent ainsi divergentes, jusqu'à la rupture définitive inscrite avec le texte L'instance de la lettre (1957) où Lacan affirme la prévalence de la parole sur le sens dans la cure.

\section{S'orienter du Réel}

\section{Politzer finalement nommé, et pourtant...}

À partir des années 1960, l'influence initiale de Politzer tend à disparaître, mais pas complètement. Ainsi, Lacan citera à nouveau le philosophe en 1961 et 1965 dans ses séminaires sur l'Identification (Séminaire IX, 1961-1962) et sur les Problèmes cruciaux pour la psychanalyse (Séminaire XII, 1965-1966) ; deux références, uniques, dans chacun de ces séminaires, pour s'y opposer. Enfin, en 1969, dans son séminaire sur L'Envers de la psychanalyse, où il cherche à dégager les ressorts du Maître capitaliste et ses effets sur le discours de l'Universitaire, Lacan convoque le philosophe révolutionnaire Politzer, quarante ans après sa thèse, pour faire de sa réflexion le discours de l'universitaire par excellence, et le " je » concret ce qui en ressort comme subjectivité.

Si Politzer est rappelé dans les années 1961-1970, c'est avant tout pour appuyer un changement majeur dans la conception de Lacan du sujet et du signifiant, en même temps qu'il introduit l'objet a dans une référence au concept de plus-value issu de la pensée de K. Marx ; un emprunt de Lacan qui marque sans nul doute sa rencontre avec Althusser. ${ }^{4}$ Pour ce dernier, il

\footnotetext{
${ }^{4}$ Rappelons qu'à la même époque, Lacan, excommunié de l'IPA, déménage son séminaire de Ste-Anne à l'École Normale Supérieure où il est désormais invité par Althusser. Un an auparavant, en 1963, le philosophe consacrait son séminaire à Lacan et à la psychanalyse (Gillot, 2009), et c'est à cette occasion que Jacques Alain Miller présentera une lecture critique de l'entreprise politzérienne ; lecture qui sera reprise par Althusser dans son texte Freud et Lacan (Althusser, 1991).
} 
revient en effet à Politzer d'avoir introduit la psychanalyse dans la réflexion philosophique (Bianco, 2016). Toutefois, c'est avant tout contre MerleauPonty et Sartre, qu'Althusser convoque le projet de Politzer pour en montrer l'impasse dans la phénoménologie. Dans la lignée de Bachelard, il dénonce la contradiction interne au concept du concret, faisant de la démarche concrète une illusion : " aucune science ne peut faire sans abstraction " (Althusser, 1997, p. 44). La CFP apparaît ainsi, pour Althusser, comme un écrit plein "d'erreurs géniales », n'ayant entraîné aucun résultat théorique, ne permettant aucunement de fonder une connaissance qui consiste justement en une abstraction des concepts, et n'ayant débouché " sur rien d'autre que sur Merleau-Ponty et Sartre » (Ibid., p. 40), à savoir sur l'existentialisme, une philosophie spiritualiste et idéologique.

Bien plus qu'à Politzer, c'est à Lacan qu'Althusser rend hommage pour sa lecture de Freud, et pour avoir fait la critique du caractère idéologique de la psychologie contemporaine, se portant contre les courants psychologiques et psychanalytiques venant des États-Unis, et leurs « techniques de réadaptation » de l'individu au sein de la société capitaliste. En effet, dans le « discours de Rome »(1956/2001b), Lacan soulignait déjà le « nouvel obscurantisme » introduit en France par Daniel Lagache sous la notion de « fonction de synthèse du moi »(Lacan, 1956/2001b, p. 143), ainsi que l'influence du behaviorisme sur la psychanalyse américaine, qui a donné naissance à la psychologie de l'Ego, toute « infléchie vers l'adaptation de l'individu à l'entourage social » (Lacan, 1956/1966c, p. 245). Des « sciences » qui, selon Althusser, reposent sur une nouvelle imposture : celle d'une « idéologie empiriste » à laquelle s'adjoint un mythe de l'unité de «l'Homme ».

\section{Le discours de l'universitaire par excellence}

En 1969, lors de son séminaire sur L'envers de la psychanalyse, Lacan invite ses auditeurs à lire Politzer dont les écrits viennent d'être réédités pour la première fois depuis 1928, en même temps qu'il souligne l'ironie de cette réédition par un éditeur universitaire sans « que l'auteur eût lui-même approuvé [...] alors que chacun sait le drame qu'a été pour lui l'accablement des fleurs sous lequel a été couvert ce qui d'abord se pose comme cri de révolte » (Lacan, 1996, p. 72). Un hommage mais aussi critique au projet du philosophe révolutionnaire, qu'il qualifiera de « discours de l'universitaire par excellence ", hommage qui se double d'une incise à l'encontre de la lecture particulière que proposent ses élèves $\mathrm{J}$. Laplanche et $\mathrm{S}$. Leclaire à propos 


\section{EPISTEMOLOGIA DA PSICOPATOLOGIA}

de l'inconscient structuré comme un langage. Or, si ce séminaire s'inscrit comme un tournant dans l'enseignement de Lacan, y dépliant le paradigme d'une " jouissance discursive », il est aussi le moment où la confiance en la primauté du langage comme élément sur lequel pourrait se fonder une science concrète du psychisme est sérieusement compromise. Nous sommes loin de cet enthousiasme qui prévalait lorsqu'il affirmait que « la psychanalyse devrait être la science du langage habité par le sujet » (Lacan, 1955-56/1981, p. 286). À ce point, il est possible pour Lacan de marquer ses différences théoriques d'avec Politzer. En effet, si Politzer, dans son retour à Freud, avait abouti à l'impasse d'une psychologie concrète fixée à la " continuité du Je », Lacan, dans ce séminaire, va dégager une position réaliste tout à fait inédite.

Avec le séminaire XVII, Lacan formalise l'écriture de quatre discours comme structures signifiantes, organisées autour de quatre places (dominante, agent ou semblant, travail, vérité, production); places qui vont être successivement occupées par quatre éléments fondamentaux de la fonction psychique $\left(\mathrm{S}_{1}, \mathrm{~S}_{2}, \$\right.$, a). Quatre places, quatre termes, quatre structures qui inscrivent ce qui fonctionne comme réalité du discours (Lacan, 197273/1975, p. 43). Le programme annoncé par Lacan de ce séminaire vise «une reprise par l'envers » (Lacan, 1996, p. 12) du projet freudien et marque un changement notable dans l'enseignement de Lacan, d'introduire à une articulation de la jouissance et du signifiant. Dès lors, si la jouissance, en tant qu'elle est interdite, fonde la structure des discours, le signifiant devient un appareil de la jouissance. C'est donc à partir de l'au-delà du principe du plaisir, de la jouissance, terme innommable de l'appareil discursif, que Lacan reprend la clinique freudienne pour introduire " à ce qui peut être démarche féconde, non pas de la pensée, mais de l'acte » (Ibid., p. 205). La jouissance apparaît comme ce qui va permettre la révolution des discours ; l'acte par lequel le discours bascule d'une forme à l'autre.

«Agent, qu'est-ce que cela veut dire ? » demande Lacan (Lacan, 1996, p. 145), en même temps qu'il relève toute l'ambiguïté de sa définition. de Libéra relèvera ainsi un premier usage de ce terme dans la philosophie anglo-saxonne du 18ème siècle, et soulignera combien la langue anglaise se construit comme une langue de l'agency permettant de « penser l'agir non plus en tant que catégorie opposée à la passion, mais en tant que disposition à l'action, disposition qui ébranle l'opposition actif/passif » (de Libéra, 2004, p. 26). Dans le domaine du droit, l'agency va dès lors permettre de décrire des modes d'action «par procuration », tandis que dans son usage politique, l'agency devient l'institution ayant une puissance d'agir pour quelqu'un. 
L'agency est le lieu effectif du pouvoir politique et l'incarnation de l'exercice du pouvoir. Dans la langue française, le terme d'agency ne sera pas sans poser quelques difficultés de traduction, si bien que ni le terme de « sujet» ni celui de « structure » ne lui correspondront totalement. Avec l'écriture des discours, Lacan disjoint l'agent du sujet, faisant du premier une place, tandis que le second s'écrit d'une lettre. Ainsi, l'agent n'est pas l'acteur mais « celui qui est fait agir » (Lacan, 1996, p. 197). Et, s'il donne son « titre » au discours, son acte ne lui appartient pas d'être déterminé par la vérité qu'il ignore.

Dans le discours de l'Universitaire, l'agent est le savoir $\left(\mathrm{S}_{2}\right)$ trouvant sa vérité du maître $\left(\mathrm{S}_{1}\right)$ refoulé sous la barre, et produisant un sujet discipliné par la mise au travail de l'objet cause du désir. Ce discours traite le réel par le savoir, mais ce savoir est déterminé par le pouvoir du signifiant maître. Dès lors, le discours du maître apparaît comme ce qui enserre « ce qui se croit révolution, ou plus exactement ce qu'on appelle romantiquement Révolution avec un grand R » (Ibid., p. 99). Autrement dit, ce qu'appelle avant tout l'acte révolutionnaire du discours de l'Universitaire, c'est un maître qui pourra régner sur lui dans une parfaite ignorance. C'est à ce point que le discours de Politzer apparaît, pour Lacan, comme « pathétique », marqué de cette position de «Unglauben », d'incroyance, de celui qui ne veut rien savoir « du coin où il s'agit de la vérité » (Ibid., p. 71).

Lacan reconnait dans la critique de Politzer « une lutte singulière » dont l'écrit témoigne, dans cette volonté d'amener la psychologie « hors du champ de tout ce qui s'est fait jusque-là » (Ibid., p. 73), en se situant au plus près de la pensée freudienne d'avoir donné une place centrale au récit dans sa psychologie concrète. Toutefois, si le récit est avant tout conçu par Politzer comme présence du « Je », pour Lacan, c'est à la « Je-cratie » qu'il retourne, à un Je qui maîtrise, par où quelque chose est identique à soi-même (Ibid., p. 74).

Lacan ne s'arrête pas là. Sur le modèle de la CFP, il va se servir de Politzer pour répondre, dans une critique cinglante, aux positions de Laplanche et Leclaire exposées lors du sixième colloque à Bonneval, et publiées sous le titre "L'inconscient, une étude psychanalytique » $(1961 / 1981) .{ }^{5}$ Ceux-ci y rendaient « hommage à un auteur » ainsi qu'à sa

${ }^{5}$ C'est au moment où Lacan se fait excommunier de l'I.P.A que Laplanche engage une critique de Politzer. Après ce séminaire, Lacan y reviendra dans Préface à une thèse en 1970, tandis que Laplanche reprendra la charge contre Politzer dans « Sens et lettre. Examen de la critique de Georges Politzer » (1981). Texte dans lequel il situe Politzer au fondement de sa divergence avec Lacan dans le contexte polémique du débat autour de l'axiome : " l'inconscient est structuré comme un langage ». 


\section{EPISTEMOLOGIA DA PSICOPATOLOGIA}

Critique qui a fait fonction «pour toute une génération (...) d'une véritable "introduction à la psychanalyse" " (Laplanche, Leclaire, 1961/1981, p. 266). Un hommage, certes, mais nuancé d'une critique. En effet, derrière la référence à Politzer, ce que visent tout particulièrement les deux psychanalystes, c'est la phénoménologie existentialiste de Merleau-Ponty et de Sartre. Avec pour second niveau : l'articulation dégagée par Lacan de l'inconscient freudien et du langage. C'est à ce point que Lacan dénonce une erreur de lecture de la part de ses deux anciens élèves. Pour ce dernier, le langage ne permet pas tant d'atteindre le réel dans l'adéquation des mots et des choses — position nominaliste - qu'il ne s'introduit dans le réel pour y créer la structure. Autrement dit, il y a du savoir dans le réel : tel est le réalisme logique de la structure impliqué dans la science. Et si le sujet de l'inconscient est le sujet de la science, alors les effets de signification sont équivalents à des réponses du réel : au réalisme logique dans la science répond un réalisme logique dans la psychanalyse qui ne conduit cependant pas à poser une réalité des universaux qui emporterait avec elle une lecture de l'inconscient comme réservoir de choses déjà là. Autrement dit, pour Lacan, il ne s'agit pas d'un réalisme de l'inconscient mais d'un réalisme de la structure.

\section{L'a-réalisme de la structure}

L'inconscient est en puissance, et ses constructions se fondent et se règlent sur le langage, toujours déjà-là mais après-coup. Ainsi, l'inconscient n'est pas condition du langage comme ont pu le soutenir Laplanche et Leclaire, bien plus, "l'inconscient est structuré comme un langage ». Si Laplanche a bien démontré combien l'inconscient ne peut s'articuler comme « je », rappelant que le sujet reçoit son message de l'Autre sous une forme inversée, si cela permet au rapporteur " de briller aux dépens de Politzer » (Lacan, 1996, p. 72), la démonstration de Laplanche n'en reste pas moins insuffisante. « La vérité parle "Je" ", un « je » qui « est peut-être innombrable », n'ayant dès lors « nul besoin de continuité du Je pour qu'il multiplie ses actes » (Lacan, 1996, p. 73). Dans Préface à une thèse, il ajoute: « A Politzer, j'eusse proposé l'image du Je innombrable, défini du seul rapport à l'unité qu'est la récurrence » (Lacan, 1970/2001c, p. 397). D'abord inscrite dans une référence au structuralisme de Saussure, la définition du signifiant se fondait sur le modèle de la différence qualitative du matériel phonique. Toutefois, à partir des années 1960, Lacan modifie sa conception et cherche à articuler le signifiant et le sujet avec la logique du non-identique à soi-même qu'il 
trouve dans le modèle numéral et arithmétique de Frege. Or, ce passage à un modèle qui se réfère à une différence absolue ne peut s'effectuer qu'au prix d'un vacillement de l'identité, par où le signifiant ne peut se signifier lui-même. Lacan va ainsi rendre logiquement possible " la constitution de l'objet à la place même de cette différence du signifiant lui-même, dans son effet subjectif » (Lacan, Leçon du 20 juin 1961). L'objet $a$ fixe le sujet en lui donnant la possibilité d'une identification, lui permettant d'échapper à la vacillation de son être.

Dès lors, s'initiant d'un « il n'y a pas d'Autre de l'Autre », d'un « il n'y a pas de métalangage », le discours de Politzer produit un «Je » concret, né de l'inconsistance de l'Autre d'y répondre en rendant une consistance au sujet dans un relayage par l'objet. Autrement dit, à l'inconsistance logique du sujet répond la consistance du $a$, du plus-de-jouir, toujours vrai. Le « Je » de Politzer apparaît ainsi, au niveau du fantasme, comme axiome du sujet, s'énonçant en une proposition logique, vraie, par hypothèse (et non pas démonstration), lui donnant l'illusion d'un tout ; un Un qui se ferme en tant que «Je » de tout un discours. Politzer se sert de la psychanalyse pour faire sa critique de la psychologie classique, en même temps qu'il cherche à en dépasser l'impasse dans la psychologie concrète. Cette impasse, il la situe au lieu même de la découverte de Freud. Politzer réfute l'hypothèse réaliste de l'inconscient, mais passe à côté du réalisme de la structure coextensive du sujet psychologique. Le Je concret vise à supprimer l'écart entre l'énoncé et l'énonciation. C'est là le point d'impasse de Politzer relevé par Lacan. C'est un « Je qui ne peut pas se dire », à moins d'emprunter la forme impersonnelle. C'est le Je d'un « je ne veux pas savoir »; il jouit, et ne le sait pas, pour pouvoir le dire. Non plus réalité du sujet, mais le sujet de la jouissance, marqué du UN de la répétition. Ainsi, le Je homogène de la psychologie concrète est un impossible, en tant que l'énoncé ne peut être équivalent à l'énonciation, sans produire un trou là où Lacan loge son objet $a$, dans la rupture de la cause empirique. Et, c'est à creuser l'écart entre signification et référence que Lacan va envisager l'objet (a) : « ce qui dévoile que le réel, en tant qu'il répond, n'est pas d'emblée donné dans son absolue transcendance réaliste, mais qu'il résulte comme tel d'une opération toujours particulière, et qu'il n'est discernable, dans l'hétérogénéité même de sa structure, que par un acte de parole dont il en est consécutivement le produit hétérogène » (Ottavi, 1997, p. 172).

Ce changement de paradigme conduira Lacan à substituer à l'antinomie de la parole et du langage, celle de l'être et du sens ; tournant réaliste d'une théorie qui s'inscrivait dans une perspective nominaliste et anti-ontologique 


\section{EPISTEMOLOGIA DA PSICOPATOLOGIA}

héritée de F. de Saussure et premier pas vers une conception qui fait valoir un rapport à l'existence hors signifiant; ce qu'il élaborera dans les années 1970.

Politzer comme Lacan cherchent à s'orienter du réel, concret, vivant. Toutefois, le réel de Lacan n'est pas le réel de Politzer. A une philosophie qu'il juge " sans matière », Politzer oppose une philosophie qui fait du genre " homme » un universel concret ; non pas un concret spéculatif mais un concret réel, et donc, particularisé, c'est-à-dire, limité, et pourtant repérable dans sa singularité. Dès lors, l'homme concret envisagé "sur le plan de l'humain », va s'inscrire comme dépassement de la définition de l'homme par la dichotomie âme/corps. L'humanité existe en tant que matière, née de la soumission. Et si, pour Politzer, c'est l'ignorance qui conduit à la soumission à l'Autre, faisant du genre homme la matière de toute aspiration au concret, la psychologie qui en découle va dès lors promouvoir un "Je » vivant « sur le plan de l'humain » (Politzer, 1926/1969a, p. 46), inscrit dans une dialectique qui « n'est créatrice que par l'acte de l'homme, qu'elle implique l'action révolutionnaire » (G. Politzer, 1926/1969a, p. 46). Si Lacan s'oriente du réel vivant c'est à partir d'une scission entre vérité et vie ; cette dernière s'inscrivant dans un matérialisme du signifiant, tandis que la dialectique du sujet et de l'Autre se corporise. Dès lors, le corps vivant n'est pas le corps symbolique, ni le corps spéculaire, mais le corps affecté de la jouissance, tandis que la perte devient l'effet structural du rapport à l'Autre. « Un sujet est ce qui peut être représenté par un signifiant pour un autre signifiant. Cela n'est-il pas calqué sur le fait que, dans ce que Marx déchiffre, à savoir la réalité économique, le sujet de la valeur d'échange est représenté auprès de la valeur d'usage? C'est dans cette faille que se produit et que choit ce qui s'appelle la plus-value. Ne compte plus à notre niveau que cette perte. Non identique désormais à lui-même, le sujet ne jouit plus »(Lacan, 2006a, p. 22). Une perte qui n'est toutefois pas à concevoir comme un effet de la domination d'un Autre ou de la lutte des classes, mais comme un « fait du discours » (Lacan, 2006a, p. 17) Ainsi, la perte apparaît comme l'effet produit de la rencontre entre le corps et l'Autre, entre le corps et la mort. Autrement dit, l'ordre symbolique, dans son incidence sur l'être parlant, sur le corps vivant, va produire un plus, une satisfaction, un plus-de-jouir, tandis que le signifiant devient cause de la jouissance. L'ignorance structurelle, relevée par Politzer, devient passion par où le sujet restaure la perte de jouissance par un désir de savoir qui appelle en même temps à une satisfaction. Le Je produit du discours de Politzer prend ainsi place de plus-de-jouir, radicalement disjoint de la 
vérité du maître ; une impuissance du savoir par où résonne le commandement de jouissance et se réalise la fonction du sujet.

\section{Conclusion}

Lecteur de Freud, Politzer met la psychanalyse au service de sa critique en même temps qu'il en fait un préliminaire à la constitution de la psychologie concrète ; une double attitude envers la psychanalyse cohérente avec son projet de reformulation intégrale de la psychologie à partir de sa critique à l'abstraction qui la déroutait, selon lui, de son destin. En premier lieu, la psychanalyse freudienne offrait à Politzer l'exemple le plus achevé d'une méthode qui engageait l'homme en situation, soit l'individu conduit par ce qui le singularisait, à savoir, son désir (Simanke, 2002, p. 178). L'interprétation de rêve en était l'exemple même de la richesse d'une future psychologie, cette fois radicalement concrète, dont les matériaux de base, soit les récits, d'une part ne dépassaient jamais l'observable, et d'autre part, ne l'amputaient non plus de sa réalité essentiellement sociale. La méthode de l'analyse freudienne des rêves était donc exemplaire pour le projet d'une psychologie concrète, sans des concepts appelant à une transcendance quelconque : « la psychanalyse, loin d'être un enrichissement de la psychologie classique, est précisément la démonstration de sa défaite » (G. Politzer, 2003, p. 23). Toutefois, en second lieu, la psychologie concrète envisagée par le philosophe ne pouvait que proscrire la métapsychologie freudienne au rang des galères de la psychologie classique. Car la métapsychologie, dès ses premiers concepts, tels, l'inconscient, proposait l'existence des réalités et d'objets qui seraient indubitablement transcendants, soit des entités métaphysiques au fond dérivés de la religion chrétienne. Ceci prouvait au philosophe que Freud n'avait pas surmonté les leurres où la psychologie classique s'était enfermée et perdue. Politzer ne va ainsi emprunter à Freud qu'une partie de sa démarche - celle méthodologique en particulier qui donne toute sa place au récit et au désir - et en même temps il va récuser l'appareil théorique de la psychanalyse en soulignant ses mystifications.

Et pourtant, la critique de Politzer touchera non seulement l'armature théorique freudienne, mais aussi la position politique de la psychanalyse, représentée à l'époque avant tout par des psychanalystes français de la première heure, de $\mathrm{A}$. Hesnard à $\mathrm{M}$. Bonaparte. Politzer en dénoncera l'esprit 


\section{EPISTEMOLOGIA DA PSICOPATOLOGIA}

scolastique de l'institution qui se marque d'une psychanalyse " dominée presqu'exclusivement par le prosélytisme », tandis que « la tactique des psychanalystes français est d'un opportunisme et par conséquent, d'une timidité excessifs » (Politzer, 1929/1969c, p. 199). En 1929, Politzer devient membre du Parti Communiste Français. La même année, il crée la Revue de Psychologie Concrète dans laquelle il expose son projet d'unification et de refondation de la psychologie en une psychologie nouvelle. La critique de Politzer est avant tout fondée sur l'objectif de démontrer le « caractère mythologique de la doctrine de la vie intérieure » (Politzer, 1929/1969b, p. 144).

Au fil des années, la position de Politzer vis-à-vis de la psychanalyse va se radicaliser. En donnant suite à ses critiques de la théorie psychanalytique, en 1933, dans son texte Psychanalyse et marxisme, Politzer réfute toute position qui se réclamerait du freudo-marxisme. Il pose une différence radicale entre la doctrine de Freud et celle de Marx, l'une idéaliste, l'autre matérialiste, et souligne que " c'est précisément pour cette raison que le freudo-marxisme n'est qu'une tentative de falsification du marxisme " (Politzer, 1933/1969d, p. 279). En 1939, dans son texte La fin de la psychanalyse, Politzer note : " La mort de Sigmund Freud replace devant notre esprit la psychanalyse qui, en fait, appartient au passé » (Politzer, 1939/1969e, p. 282). Plus qu'une critique exclusivement théorique, Politzer en dénonce sa dérive au plan politique, critiquant la psychanalyse d'être « tombée sous l'influence de courants idéologiques rétrogrades » (G. Politzer, 1939/1969e, p. 301), et d'être devenue un outil au service de la bourgeoisie, négociant avec le nazisme. Une position radicale de Politzer qui aura pour conséquence une introduction tardive du freudo-marxisme en France ; les travaux de Reich et Marcuse restant quasiment inconnus jusque dans les années 1960. L'accueil et le destin du projet concret de Politzer dans la pensée du 20e siècle apparaît ainsi paradoxal. En effet, malgré sa critique, violente, de la psychologie classique en général, de la métapsychologie freudienne et de l'institution psychanalytique en particulier, les idées de Politzer vont dépasser le milieu proprement psychanalytique et marqueront la plupart des discussions de la scène intellectuelle française (Prado Jr., 1991, pp. 14-18). Ainsi, la CFP comme présentation critique de l'œuvre freudienne va intéresser et influencer au moins trois générations de penseurs français, tels que Merleau-Ponty, Sartre, dans les années 1940, et plus tard, Leclaire et Pontalis, Paul Ricœur, Daniel Widlocher (Furlan, 1999, p. 118). Au contraire de Politzer, pour Lacan, il n'y a pas de réalité des Universaux. Ainsi, la formule " il existe un homme » n'est pas inscriptible dans la structure, celle-ci soutenant son 
existence d'une exception. Autrement dit, l'homme est un possible du fait de l'universel, mais, cet universel ne lui assure aucune existence, renvoyant cette dernière au niveau du particulier. Lacan va ainsi prendre position, en indiquant les voies d'un dépassement possible de la querelle dite " des Universaux », en dégageant une position en creux, par où se noue la logique pure du sujet au corps affecté de jouissance. Ainsi, «c'est bien à la psychanalyse seule, et au long des élaborations de Lacan, que revient le mérite d'avoir authentiquement résolu, d'avoir épistémologiquement dépassé le cadre disputatif de la pensée occidentale, fixé depuis si longtemps par la tension entre Nominalisme et Réalisme, et qui a permis précisément le moment cartésien de l'émergence de la science, avec le cortège de questions qui se déploient alors » (Ottavi, 1997, p. 161). Si la pensée de Politzer va influencer de nombreux intellectuels, à la fois dans le champ de la philosophie et de la psychologie, il va toutefois se maintenir comme une référence discrète, voir est passée sous silence (LantériLaura, 1968). C'est en filigrane de l'épistémologie que s'inscrit Politzer. Et s'il en ressort comme "fantôme", "c'est dans le nominalisme essentiel à l'Université moderne, soit celle dont s'enfume le capitalisme", que Lacan lit "l'échec scandaleux" de la critique de Politzer (Lacan, 1970/2001c, p. 397). Un nominalisme de l'université par où le sujet se réduit à ce qui peut s'en calculer, s'étiqueter et se ranger dans l'ordonnance d'une classification.

\section{References}

Alberti C. (2020, mars). Rien de plus concret que le rêve, son usage, son interprétation. Tu rêves encore?, La Cause du désir, 104.

Althusser, L. (1991). Freud e Lacan - Marx e Freud. Rio de Janeiro, RJ: Graal

Althusser, L. (1997). Freud et Lacan. In Écrits sur la psychanalyse : Freud et Lacan. Paris, FR: IMEC/Stock.

Askofaré, S., Gaspard, J.-L., Macary-Garipuy, P., \& Sauret, M.-J. (2008, dez.). Une physique du sens ? Le problème de la formalisation en psychanalyse », Revista Latinoamericana de Psicopatologia Fundamental (Brésil), XI(4), 573-587.

Basso E. (2016). Foucault entre psychanalyse et psychiatrie ' Reprendre la folie au niveau de son langage". Archives de Philosophie, 1(79), 27- 54. https://www.cairn.info/revue-archives-de-philosophie-2016-1-page-27.htm 


\section{EPISTEMOLOGIA DA PSICOPATOLOGIA}

Bianco G.. La signification du concret. Psychologie, philosophie et politique chez Georges Politzer. Paris, FR: Hermann.

Bruyeron R., Roudinesco E., \& Worms F. (2013). Pourquoi Georges Politzer estil toujours actuel ? Table Ronde, Entretiens réalisés par B. Nolan, Journal l'Humanité, 17 mai 2013. https://www.humanite.fr/debats/pourquoi-georgespolitzer-est-il-toujours-actuel-t-541759.

de Libéra A. (2004). Agency. In Vocabulaire européen des philosophies (sous la direction de B. Cassin), Paris, FR: Seuil/Le Robert.

Freud, S. (2003). L'interprétation du rêve (Vol. IV). Paris, FR: PUF. coll. « Euvres complètes ». (Travail original publié en 1900).

Freud, S., Breuer J. (1996). Études sur l'hystérie. Paris, FR: PUF, 1996. (Travail original publié en 1893-1895).

Furlan, R. (1999). Freud, Politzer, Merleau-Ponty. Psicologia USP, 10(2), 117-138.

Galhardo P. (2016, dez.). Merleau-Ponty avec et contre Politzer : le sens et la force dans la psychanalyse freudienne. doispontos : Curitiba, São Carlos, 13(3), 177-186.

Gillot, P. (2009). Althusser et la psychanalyse. Paris, FR: PUF.

Lacan, J. (1961-62). Le séminaire. Livre IX. L'Identification. Inédit.

Lacan, J. (1965-66). Le séminaire. Livre XII. Problèmes cruciaux pour la psychanalyse. Inédit.

Lacan, J. (1966a). Au-delà du principe de réalité. In Écrits. Paris, FR: Seuil. (Travail original publié en 1936).

Lacan, J. (1966b). Propos sur la causalité psychique. In Écrits. Paris, FR: Seuil. (Travail original publié en 1946).

Lacan, J. (1966c). Fonction et champ de la parole et du langage en psychanalyse. In Écrits (p. 245). Paris, FR: Seuil. (Travail original publié en 1956).

Lacan, J. (1966d). À la mémoire d'Ernest Jones : sur la théorie du symbolisme, suivi de “D'un syllabaire après-coup". In Écrits. Paris, FR: Seuil. (Travail original publié en 1959).

Lacan, J. (1975). Le Séminaire. Livre XX. Encore. Paris, FR:Seuil. (Travail original publié en 1972-73).

Lacan, J. (1981). Le séminaire. Livre III. Les psychoses. Paris, FR: Seuil. (Travail original publié en 1955-56).

Lacan, J. (1994). Le séminaire. Livre IV. La relation d'objet. Paris, FR: Seuil. (Travail original publié en 1956-57).

Lacan, J. (1996). Le séminaire. Livre XVII. L'Envers de la psychanalyse. Paris, FR: Seuil. (Travail original publié en 1969-70).

Lacan J. (1998). La psychose paranoïaque et ses rapports avec la personnalité. Paris, FR: Seuil. (Travail original publié en 1932). 
Lacan, J. (2001a). Les complexes familiaux dans la formation de l'individu. In Autres écrits. Paris, FR: Seuil. (Travail original publié en 1938).

Lacan, J. (2001b). Discours de Rome. In Autres écrits. Paris, FR: Seuil. (Travail original publié en 1956).

Lacan J. (2001c). Préface à une thèse. In Autres écrits. Paris, FR: Seuil. (Travail original publié en 1970).

Lacan, J. (2006a). Le séminaire. Livre XVI. D'un Autre à l'autre. Paris, FR: Seuil. (Travail original publié en 1968-69).

Lacan, J. (2006b). Le Séminaire. Livre XVIII. D’un discours qui ne serait pas du semblant. Paris, FR: Seuil, 2006. (Travail original publié en 1970-71).

Lanteri-Laura G. (1968). Nizan et Politzer quarante ans après. Critique, 255-256, 772-789

Laplanche, J. (1981). Sens et lettre. Examen de la critique de Georges Politzer. In Problématiques IV. L'inconscient et le ça. Paris, FR: PUF.

Laplanche J., \& Leclaire S. (1981). L'inconscient, une étude psychanalytique. In Laplanche J., Problématiques IV. L'Inconscient et le ça (pp. 261-321). Paris, FR: PUF.

Merleau-Ponty, M. (2013). La structutre du comportement. Paris, FR: PUF. (Travail original publié en 1942)

Ottavi, L. (1997). Psychanalyse, réalité et science; quelques repérages d'un temps de la problématique chez Lacan. Les sites de l'exil.

Politzer, G. (2003). Critique des fondements de la psychologie. Paris, FR: PUF (Quadrige).

Politzer, G. (1969a). Introduction. In Écrits 1: La philosophie et les mythes. Paris, FR: Éditions sociales. (Travail original publié en 1926).

Politzer, G. (1969b). Les fondements de la psychologie. In Écrits 2: Les fondements de la psychologie. Paris, FR: Éditions sociales. (Travail original publié en 1929).

Politzer, G. (1969c). La crise de la psychanalyse. In Écrits 2: Les fondements de la psychologie. Paris, FR: Éditions sociales. (Travail original publié en 1929).

Politzer, G. (1969d). Psychanalyse et marxisme : Un faux contre-révolutionnaire, le freudo-marxisme. In Écrits 2: Les fondements de la psychologie. Paris, FR: Éditions sociales. (Travail original publié en 1933).

Politzer, G. (1969e). La fin de la psychanalyse. In Écrits 2: Les fondements de la psychologie. Paris, FR: Éditions sociales. (Travail original publié en 1939).

Prado Jr., B. (1991). Georges Politzer e os sessenta anos da 'Crítica aos fundamentos da psicologia'”. In B. Prado Jr., L. R. Monzani, O. Gabbi Jr. (Orgs), Filosofia da Psicanálise. São Paulo, SP: Brasiliense.

Simanke, R. T. (2002). Metapsicologia lacaniana: os anos de formação. São Paulo, SP: Discurso Editorial. 


\section{EPISTEMOLOGIA DA PSICOPATOLOGIA}

\section{Resumos}

(De G. Politzer e sua crítica ao inconsciente de Freud ao a-realismo de J. Lacan)

Em 1928, em sua Crítica aos Fundamentos da Psicologia, G. Politzer apresentou as características de uma psicologia que visava reformar a psicologia experimental universitária. Partindo da teoria dos sonhos de Freud, Politzer abriu uma concepção de psicologia nova em seu tempo. Suas obras, que Lacan utilizará em sua tese para abordar o fenômeno paranóico, participaram de um violento debate dentro do movimento comunista dividido entre anti-Freudianismo e Freudo-Marxismo. Ao dar um lugar essencial à fala, à narrativa, Politzer reivindicou uma psicologia concreta. A sua recusa da doutrina freudiana, segundo ele dependente da tradição metafísica da psicologia, incluía também a hipótese do inconsciente numa crítica severa do realismo, uma inclusão que nunca foi aceita por Lacan, que lhe opôs a premissa de que o inconsciente é estruturado como uma linguagem. Esta orientação imporia logicamente um realismo de estrutura que se articula, por sua vez, com a falta da causa empírica.

Palavras-chave: Psicologia concreta, inconsciente, realismo, objeto a

(From G. Politzer and his critique of Freud's unconscious to J. Lacan's a-realism)

In 1928, in his Critique of the Foundations of Psychology, G. Politzer presented the features of a psychology aimed at reformulating academic experimental psychology. Based on Freud's theory of dreams, Politzer presents a concept of psychology that was completely new at that time. Lacan drew inspiration from his work for his thesis on paranoia and took part in a violent debate by the communist movement, which was divided between anti-Freudianism and Freudo-Marxism. By giving an essential place to speech, to narrative, Politzer claimed a concrete psychology. His refusal of Freud's doctrine, which he thought depended on the metaphysical tradition of psychology, also included the hypothesis of the unconscious in a severe critique of realism, an inclusion which Lacan rejected and opposed with the premise that the unconscious is structured like a language. This orientation would logically impose a realism of structure that is related, in turn, to the lack of the empirical cause.

Key words: Concrete psychology, unconscious, realism, object a

(De G. Politzer y su critica al inconsciente de Freud al a-realismo de J. Lacan):

En 1928, en su Crítica de los Fundamentos de la Psicología, G. Politzer presentó las características de una psicología que pretendía reformar la psicología experimental universitaria. Partiendo de la teoría de los sueños de Freud, Politzer 
presentó una concepción de psicología novedosa para su época. Sus trabajos, que Lacan utilizará en su tesis para abordar el fenómeno de la paranoia, formaron parte de un debate violento dentro del movimiento comunista dividido entre en antifreudianismo y el freudomarxismo. Al dar un lugar esencial al discurso, a la narrativa, Politzer reivindicó una psicología concreta. Su rechazo a la doctrina freudiana, que según él dependía de la tradición metafísica de la psicología, incluía también la hipótesis del inconsciente en una crítica severa del realismo, una inclusión que nunca sería aceptada por Lacan, que se oponía a ella con la premisa de que el inconsciente es estructurado como un lenguaje. Esta orientación impondría lógicamente un realismo de la estructura que se articula, a su vez, a la falla de la causa empírica.

Palabras clave: Psicología concreta, inconsciente, realismo, objeto a

Citação/Citation: Marx, M., Silva Junior, N., \& Gaspard, J.-L. (2021, março). De G. Politzer et sa critique de l'inconscient de Freud à l'a-réalisme de J. Lacan. Revista Latinoamericana de Psicopatologia Fundamental, 24(1), 140-163. http://dx.doi.org/10.1590/1415-4714.2021v$24 \mathrm{n} 1 \mathrm{p} 140.8$.

Editoras/Editors: Prof. Dr. Claudio E. M. Banzato

Submetido/Submitted: 17.6.2020 / 16.17.2020 Revisado/Revised: 29.10 .2020 / 10.29.2020

Aceito/Acepted: $2.11 .2020 / 11.2 .2020$

Copyright: (C) 2009 Associação Universitária de Pesquisa em Psicopatologia Fundamental/ University Association for Research in Fundamental Psychopathology. Este é um artigo de livre acesso, que permite uso irrestrito, distribuição e reprodução em qualquer meio, desde que o autor e a fonte sejam citados / This is an open-access article, which permits unrestricted use, distribution, and reproduction in any medium, provided the original authors and sources are credited.

Financiamento/Funding: Este trabalho não recebeu apoio / This work received no funding.

Conflito de interesses/Conflict of interest: Os autores declaram que não há conflito de interesses. / The authors declare that there is no conflict of interest. 


\section{EPISTEMOLOGIA DA PSICOPATOLOGIA}

Mélinda Marx

Psychologue; Psychanalyste; Docteure en Psychopathologie

Laboratoire EA4050

Université Rennes 2, France

m.marx1277@gmail.com

https://orcid.org/0000-0001-9116-9490

Nelson da Silva Junior

Psychanalyste; Professeur de l'Université de São Paulo

Laboratoire de Théorie Sociale, Philosophie et Psychanalyse

nelsonsj1961@gmail.com

https://orcid.org/0000-0002-2454-5019

JeAn-LuC Gaspard

Psychanalyste; Professeur des Universités en Psychopathologie

Laboratoire EA4050

Université Rennes 2, France

jeanlucgaspard@gmail.com

https://orcid.org/0000-0002-8639-3487

This is an open-access article, which permits unrestricted use, distri- 